\title{
On $C_{0}$. multi-contractions having a regular dilation
}

by

\author{
DAN PopovicI (Timişoara)
}

\begin{abstract}
Commuting multi-contractions of class $C_{0}$. and having a regular isometric dilation are studied. We prove that a polydisc contraction of class $C_{0}$. is the restriction of a backwards multi-shift to an invariant subspace, extending a particular case of a result by R. E. Curto and F.-H. Vasilescu. A new condition on a commuting multi-operator, which is equivalent to the existence of a regular isometric dilation and improves a recent result of $\mathrm{A}$. Olofsson, is obtained as a consequence.
\end{abstract}

1. Introduction. The problem of finding an isometric (or a unitary) dilation of a commuting system of contractions was proposed by Sz.-Nagy [8] in the early fifties: for a given commuting system $T=\left(T_{1}, \ldots, T_{n}\right)$ of bounded operators (called commuting multi-operator; briefly c.m.) on a Hilbert space $\mathscr{H}$ find conditions for the existence of a c.m. $V=\left(V_{1}, \ldots, V_{n}\right)$ consisting of isometric operators (or, equivalently [5], unitary operators) on a Hilbert space $\mathscr{K}$ containing $\mathscr{H}$ (as a closed subspace) such that

$$
T^{m} h=P_{\mathscr{H}} V^{m} h, \quad m \in \mathbb{Z}_{+}^{n}, h \in \mathscr{H}
$$

(if $T=\left(T_{1}, \ldots, T_{n}\right)$ and $m=\left(m_{1}, \ldots, m_{n}\right) \in \mathbb{Z}_{+}^{n}$ we use the notation $T^{m}=\prod_{i=1}^{n} T_{i}^{m_{i}} ; P_{\mathscr{H}}$ is just the orthogonal projection of $\mathscr{K}$ onto $\left.\mathscr{H}\right)$. Then $V$ is said to be an isometric (respectively a unitary) dilation of $T$. Ando [1] proved that arbitrary pairs of commuting contractions have isometric dilations. Unfortunately, according to a counter-example given by Parrott [7], the existence is not always ensured for systems of at least three commuting contractions. By contrast to the single operator case, for $n \geq 2$, the minimality condition

$$
\mathscr{K}=\bigvee_{m \in \mathbb{Z}_{+}^{n}} V^{m} \mathscr{H}
$$

does not ensure the uniqueness, up to unitary equivalence, of the isometric dilation $V$ (if such a dilation does exist). However, if $V$ is a minimal isometric

2000 Mathematics Subject Classification: 47A20, 47A13, 47A45.

Key words and phrases: regular isometric dilation, commuting multi-contraction, polydisc contraction, class $C_{0}$. 
dilation of $T$ then $\mathscr{H}$ is invariant under $V^{*}:=\left(V_{1}^{*}, \ldots, V_{n}^{*}\right)$ and $\left.V_{i}^{*}\right|_{\mathscr{H}}=T_{i}^{*}$, $i=1, \ldots, n: V^{*}$ is said to be a minimal co-isometric extension of $T^{*}$. In addition, if $U$ is the minimal unitary extension of $V$ then the restriction of $U^{*}$ to $\bigvee_{m \in \mathbb{Z}_{+}^{n}} U^{* m} \mathscr{H}$ is a minimal isometric dilation of $T^{*}$. It will be referred to as the minimal isometric dilation of $T^{*}$ corresponding to $V$.

It was the idea of Brehmer [2] to introduce a special class of isometric or unitary dilations: an isometric or unitary dilation $V$ (on $\mathscr{K}$ ) of $T$ (on $\mathscr{H}$ ) is called regular if it satisfies

$$
\left(T^{m^{-}}\right)^{*} T^{m^{+}} h=P_{\mathscr{H}}\left(V^{m^{-}}\right)^{*} V^{m^{+}} h, \quad m \in \mathbb{Z}^{n}, h \in \mathscr{H} .
$$

Brehmer provided necessary and sufficient conditions on $T$ ensuring the existence of a regular isometric (hence also unitary) dilation, namely,

$$
\Delta_{T}^{\beta}:=\sum_{0 \leq \alpha \leq \beta}(-1)^{|\alpha|} T^{* \alpha} T^{\alpha} \geq 0
$$

for every $\beta \in \mathbb{Z}_{+}^{n}$ with $\beta \leq e:=(1, \ldots, 1)$. If it exists, a minimal regular isometric dilation is unique up to unitary equivalence. A c.m. $T$ is said to be a polydisc contraction if $\Delta_{T}^{e} \geq 0$. If $T$ is a commuting multi-contraction with $\Delta_{T}^{e}=0$ then $T$ is a polydisc isometry $([3])$.

For a vector $r=\left(r_{1}, \ldots, r_{n}\right) \in \mathbb{R}^{n}$ and a c.m. $T=\left(T_{1}, \ldots, T_{n}\right)$ let $r T:=\left(r_{1} T_{1}, \ldots, r_{n} T_{n}\right)$. It is not hard to observe that, for any multi-index $\beta=\left(\beta_{1}, \ldots, \beta_{n}\right)$ with $0 \leq \beta \leq e$,

$$
\Delta_{r T}^{\beta}=\Delta_{T}^{\beta}+\sum_{k=1}^{n} \sum_{i_{1}, \ldots, i_{k}=1}^{n}\left(\prod_{p=1}^{k} \beta_{i_{p}}\left(1-r_{i_{p}}^{2}\right) T_{i_{p}}^{*}\right) \Delta_{T}^{\beta-\sum_{p=1}^{k} \beta_{i_{p}} e_{i_{p}}}\left(\prod_{p=1}^{k} T_{i_{p}}\right) .
$$

If $\beta$ is fixed and $\Delta_{T}^{\alpha} \geq 0$ for every $\alpha$ with $0 \leq \alpha \leq \beta$, we deduce that

$$
\Delta_{r T}^{\alpha} \geq \Delta_{T}^{\alpha}, \quad 0 \leq \alpha \leq \beta,-e \leq r \leq e .
$$

The following result, which can be seen as a particular case of [3, Lemma 3.6] (it was recently rediscovered in [6, Proposition 2.1]), is then obtained:

Proposition 1.1. If $T$ has a regular isometric dilation then so does $r T$ for every vector $r$ with $-e \leq r \leq e$.

It is our aim in the following to study c.m. of class $C_{0}$., namely the class of c.m. $T=\left(T_{1}, \ldots, T_{n}\right)$ which satisfy $T_{i}^{k} h \stackrel{k}{\rightarrow} 0(h \in \mathscr{H}, i=1, \ldots, n) . T$ is of class $C_{.0}$ if $T^{*}$ belongs to the class $C_{0}$..

For a c.m. of class $C_{0}$. only one of the Brehmer positivity conditions (2) must a priori be satisfied in order to obtain a regular isometric dilation. More precisely, extending a particular case of a result by R. E. Curto and F.-H. Vasilescu [3, Theorem 3.16], we prove that a polydisc contraction of class $C_{0}$. is the restriction of a backwards multishift to an invariant subspace (Theorem 2.1). Improving a recent result by A. Olofsson [6] (Corollary 2.5), 
we deduce a new necessary and sufficient condition on a c.m. in order to ensure the existence of a regular isometric dilation (Corollary 2.4). By contrast to the methods in [6] (where powerful tools of measure theory are used), our proofs are drastically simplified by applying (only) standard operator theory.

2. $C_{0}$. multi-contractions and dilations. Recall from [3] that a multishift is just a doubly commuting tuple of shifts; a backwards multi-shift is the adjoint of a multi-shift.

Polydisc contractions of class $C_{0}$. are restrictions of backwards multishifts. More precisely, extending a particular case of a result by R. E. Curto and F.-H. Vasilescu [3, Theorem 3.16], we have:

Theorem 2.1. Let $T=\left(T_{1}, \ldots, T_{n}\right)$ be a c.m. on $\mathscr{H}$. The following conditions are equivalent:

(i) $T$ is a polydisc contraction of class $C_{0}$.;

(ii) $T$ is the restriction of a backwards multi-shift to an invariant subspace;

(iii) $T$ has a (minimal) regular isometric dilation and the corresponding minimal isometric dilation of $T^{*}$ is a multi-shift.

Proof. Let $m(1 \leq m \leq n)$ be a fixed positive integer and suppose that $\Delta_{T}^{\beta} \geq 0$ for every $\beta=\left(\beta_{1}, \ldots, \beta_{n}\right) \in \mathbb{Z}_{+}^{n}$ with $\beta \leq e$ and $|\beta|:=\beta_{1}+\cdots+\beta_{n}$ $=m$.

Easy computations show that

$$
\Delta_{T}^{\alpha}-T_{i}^{*} \Delta_{T}^{\alpha} T_{i}=\Delta_{T}^{\alpha+e_{i}}
$$

for any $\alpha \in \mathbb{Z}_{+}^{n}$ with $\alpha \leq e$ and $|\alpha|=m-1$ and any $i \in\{1, \ldots, n\} \backslash \operatorname{supp} \alpha$. Then

$$
T_{i}^{*} \Delta_{T}^{\alpha} T_{i} \leq \Delta_{T}^{\alpha}
$$

since $0 \leq \alpha+e_{i} \leq e$ and $\left|\alpha+e_{i}\right|=m$. By iteration we deduce that

$$
T_{i}^{* p} \Delta_{T}^{\alpha} T_{i}^{p} \leq \Delta_{T}^{\alpha} \quad \text { for every } p \in \mathbb{Z}_{+} .
$$

If (i) holds true then

$$
\left|\left\langle T_{i}^{* p} \Delta_{T}^{\alpha} T_{i}^{p} h, h\right\rangle\right| \leq\left\|\Delta_{T}^{\alpha}\right\|\left\|T_{i}^{p} h\right\|^{2} \stackrel{p}{\rightarrow} 0
$$

for every $h \in \mathscr{H}$. Hence $\Delta_{T}^{\alpha} \geq 0$ by (3). We proceed inductively to prove that a polydisc contraction of class $C_{0}$. has a regular isometric dilation. The corresponding minimal isometric dilation $V=\left(V_{1}, \ldots, V_{n}\right)$ of $T^{*}$ is then doubly commuting ([4], [9]). Moreover,

$$
\left\|V_{i}^{* p} V^{m} h\right\|=\left\|V^{m} V_{i}^{* p} h\right\|=\left\|V_{i}^{* p} h\right\|=\left\|T_{i}^{p} h\right\| \stackrel{p}{\rightarrow} 0
$$

for every $i \in\{1, \ldots, n\}, m \in \mathbb{Z}_{+}^{n}$ and $h \in \mathscr{H}$. The minimality condition (1) 
forces $V_{i} \in C_{\text {. } 0}$ for any $i$. Therefore $V$ is a multi-shift, and this completes the proof of (i) $\Rightarrow$ (iii).

If $V$ is the minimal isometric dilation of $T^{*}$ given by (iii) (corresponding to the minimal regular isometric dilation of $T$ ) then $V^{*}$ (which is a backwards multi-shift) is a minimal co-isometric extension of $\left(T^{*}\right)^{*}=T$. Condition (ii) is obtained.

Finally, observe that a backwards multi-shift is of class $C_{0}$. and has a regular dilation (being doubly commuting). The same is then true for its restriction to an invariant subspace. The implication (ii) $\Rightarrow$ (i) is proved.

The restriction of a c.m. consisting of backwards shifts to an invariant subspace obviously belongs to the class $C_{0}$., but it is not necessarily a polydisc contraction:

EXAmPLE 2.2. Let $S$ be the standard (unilateral) shift on $\ell_{\mathbb{Z}_{+}}^{2}$, that is,

$$
S\left(c_{0}, c_{1}, \ldots\right)=\left(0, c_{0}, c_{1}, \ldots\right), \quad\left(c_{p}\right)_{p \geq 0} \in \ell_{\mathbb{Z}_{+}}^{2} .
$$

Then $T=\left(S^{*}, \ldots, S^{*}\right)$ is a c.m. on $\ell_{\mathbb{Z}_{+}}^{2}$ consisting of backwards shifts. In addition, if $B$ is the standard bilateral shift on $\ell_{\mathbb{Z}}^{2}$, namely

$$
B\left(c_{p}\right)_{p \in \mathbb{Z}}=\left(c_{p-1}\right)_{p \in \mathbb{Z}}, \quad\left(c_{p}\right)_{p \in \mathbb{Z}} \in \ell_{\mathbb{Z}}^{2},
$$

then, under the obvious identification $\ell_{\mathbb{Z}_{+}}^{2} \subset \ell_{\mathbb{Z}}^{2}, U=\left(B^{*}, \ldots, B^{*}\right)$ is a unitary dilation of $T$. However, $T$ does not have a regular isometric dilation (equivalently, by Theorem 2.1, $T$ is not a polydisc contraction). We only have to observe that

$$
\Delta_{T}^{e_{1}+e_{2}}=I-2 S S^{*}+S^{2} S^{* 2}
$$

fails to be a positive operator since, for example,

$$
\left\langle\Delta_{T}^{e_{1}+e_{2}}(0,1,0, \ldots),(0,1,0, \ldots)\right\rangle=-1 .
$$

Corollary 2.3. A polydisc contraction consisting of strict contractions has a regular isometric dilation.

A new condition on a c.m. $T$, equivalent to the existence of a regular isometric dilation, is also obtained:

COROLlaRY 2.4. T has a regular isometric dilation if and only if there exists a sequence $r_{k} \in \mathbb{R}_{+}^{n}, r_{k} \leq e$, such that $r_{k} \rightarrow e$ as $k \rightarrow \infty$ and $r_{k} T$ is a polydisc contraction of class $C_{0}$. for all $k$.

Proof. If $T$ has a regular isometric dilation then so does $r T$ for every $r \in \mathbb{R}_{+}^{n}$ with $r \leq e$ (by Proposition 1.1).

Conversely, if $r_{k} T$ is a polydisc contraction of class $C_{0}$. then, by Theorem $2.1, r_{k} T$ has a regular isometric dilation. Since $r_{k} \rightarrow e$ as $k \rightarrow \infty$ we conclude that $T$ has a regular isometric dilation. 
In particular, our methods provide a result of Olofsson [6, Theorem 2.1]:

Corollary 2.5. Let $T$ be a c.m. consisting of contractions. Then $T$ has a regular isometric dilation if and only if there exists a sequence $r_{k}=$ $\left(r_{1}^{k}, \ldots, r_{n}^{k}\right) \in \mathbb{R}_{+}^{n}, r_{i}^{k}<1$, such that $r_{k} \rightarrow e$ as $k \rightarrow \infty$ and $r_{k} T$ is a polydisc contraction for all $k$.

A similar argument can be applied for polydisc isometries:

Proposition 2.6. A c.m. of class $C_{0}$. on a non-null Hilbert space cannot be a polydisc isometry.

Proof. Let $T=\left(T_{1}, \ldots, T_{n}\right)$ be a c.m. of class $C_{0}$. on a Hilbert space $\mathscr{H}$. Suppose that $T$ is a polydisc isometry.

Proceed as in the proof of Theorem $2.1(\mathrm{i}) \Rightarrow$ (iii). Suppose that $\Delta_{T}^{\beta}=0$, for every $\beta \in \mathbb{Z}_{+}^{n}$ with $\beta \leq e$ and $|\beta|=m$ ( $m$ is fixed; $1 \leq m \leq n$ ). Then, for any $\alpha \in \mathbb{Z}_{+}^{n}$ with $\alpha \leq e$ and $|\alpha|=m-1$, and for all $i \in\{1, \ldots, n\} \backslash \operatorname{supp} \alpha$ and $p \in \mathbb{Z}_{+}$,

$$
T_{i}^{* p} \Delta_{T}^{\alpha} T_{i}^{p}=\Delta_{T}^{\alpha}
$$

We let $p \rightarrow \infty$ in (4) to deduce that $\Delta_{T}^{\alpha}=0$. We repeat this step inductively to obtain

$$
\Delta_{T}^{e_{i}}=I-T_{i}^{*} T_{i}=0, \quad i \in\{1, \ldots, n\} .
$$

Hence each $T_{i}$ is isometric, a contradiction.

COROLlary 2.7. There are no polydisc isometries consisting of strict contractions.

\section{References}

[1] T. Ando, On a pair of commutative contractions, Acta Sci. Math. (Szeged) 24 (1963), 88-90.

[2] S. Brehmer, Über vertauschbare Kontraktionen des Hilbertschen Raumes, ibid. 22 (1961), 106-111.

[3] R. E. Curto and F.-H. Vasilescu, Standard operator models in the polydisc, Indiana Univ. Math. J. 42 (1993), 791-810.

[4] D. Gaşpar and N. Suciu, On the intertwinings of regular dilations, Ann. Polon. Math. 66 (1997), 105-121.

[5] T. Itô, On the commutative family of subnormal operators, J. Fac. Sci. Hokkaido 58 (1958), 1-5.

[6] A. Olofsson, Operator valued n-harmonic measure in the polydisc, Studia Math. 163 (2004), 203-216.

[7] S. Parrott, Unitary dilations for commuting contractions, Pacific J. Math. 34 (1970), 481-490.

[8] B. Sz.-Nagy, Transformations de l'espace de Hilbert, fonctions de type positif sur un groupe, Acta Sci. Math. (Szeged) 15 (1953), 104-114. 
[9] D. Timotin, Regular dilations and models for multi-contractions, Indiana Univ. Math. J. 47 (1998), 671-684.

Department of Mathematics and Computer Science

University of the West Timişoara

Bd. Vasile Pârvan nr. 4

RO-300223 Timişoara, Romania

E-mail: popovici@math.uvt.ro

Received January 17, 2005

Revised version May 10, 2005 